\title{
EXELFS AND EXAFS: COMPLEMENTARY PROBES INTO THE STRUCTURE OF METALLIC GLASSES
}

\author{
Faisal M. Alamgir, Himanshu Jain, David B. Williams and Gilles Hug* \\ Department of Materials Science \& Engr., Lehigh University, Bethlehem, PA, USA \\ * ONERA, Chatillon Cedex, France
}

Extended x-ray absorption fine structure (EXAFS) analysis is a very powerful technique for obtaining structural information from materials in which there are strong local atomic correlations that govern the properties of the overall system, such as in glasses. Furthermore, EXAFS is atom specific and therefore, even the most complex multi-component structures have the potential of being solved if the information around all the atoms can be accessed. There are, however, significant limitations of the conventional EXAFS. To begin with, accessing the K-edges below 3 $\mathrm{keV}$ becomes problematic since absorption at these energies requires sub-micron sample thickness for transmission EXAFS and very few beamlines are available to do fluorescence EXAFS. Also, the spatial resolution of X-rays is not high enough to study sub-micron scale phases.

The electron analogue of EXAFS is the extended electron energy loss fine structure (EXAFS) within electron energy-loss spectroscopy (EELS) in a transmission electron micioscope (TEM). Since the incident electrons in a TEM can be focused, EXELFS offers the unique ability to obtain atomic and electronic structure on a nanometer-scale spatial resolution. Further, since TEMs operate in high vacuum and use thin specimens, EELS is especially suited to K-edge analysis of low atomic number elements that EXAFS. As do the synchrotron X-rays in EXAFS, the incident electron beam in a TEM ionizes an atom and the resulting ejected electrons create modulations in the conduction band as they scatter back from neighboring atoms. These modulations are represented as a function $\chi(\boldsymbol{k})$, where $\boldsymbol{k}$ is in wavenumbers. The Fourier transform of a k-weighted $\chi(\boldsymbol{k}), \mathrm{FT}\left[\mathrm{k}^{\mathrm{n}} \cdot \chi(\boldsymbol{k})\right]$, is proportional to probability of finding an atom at a given radial distance from the ionized atom.

The local structure in a prototypical bulk metallic glass (BMG), the Pd-Ni-P system was studied using EXELFS and EXAFS, where the former provided structural information around $\mathrm{P}$ (where EXAFS is difficult) and the EXAFS provided information around the transition metals (where EXELFS is problematic). The environment around all the constituent elements in this BMG system was thus accessible, revealing the positions, their types and the level of static disorder around each constituent element. The importance of each element to the glass-forming ability of this BMG system could thus be examined in terms of these structural parameters. For example, the difference between the peak positions of the magnitude and the imaginary parts of FT[k $\cdot ?(\mathrm{k})]$ is related to the phase change of the electron as it is backscattered from its nearest neighbor. A change in this phase is observe that crystallization of $\mathrm{Pd}_{30} \mathrm{Ni}_{50} \mathrm{P}_{20}$, indicating that it involves a change in the nearest neighbor of $\mathrm{P}$ (Figure 1) with the likely replacement of Ni by Pd upon crystallization. This change in the chemical short-range order is a measure of the ease of crystallization, or the lack of glass stability. It was further observed from the environment around the other elements, that the Ni atoms are relatively free to rearrange themselves as the glass crystallizes while the Pd atoms seem more bound in place. It was found that the compositions which mark the ends of the bulk glass formation range are well defined in terms of the structures calculated by FEFF7 [i] an ab initio code, for the crystal binary alloys $\mathrm{Pd}_{3} \mathrm{P}$ and $\mathrm{Ni}_{3} \mathrm{P}$ (Figure 2) and the mixing of the two types of structure stabilizes 
the intermediate glass composition, the $\mathrm{Pd}_{40} \mathrm{Ni}_{40} \mathrm{P}_{20}$, by introducing greater kinetic hindrance to crystallization.

i S. I. Zabinsky, J. J. Rehr, A. Ankudinov, R. C. Albers, and M. J. Eller, Phys. Rev. B, 52, 2995 (1995).

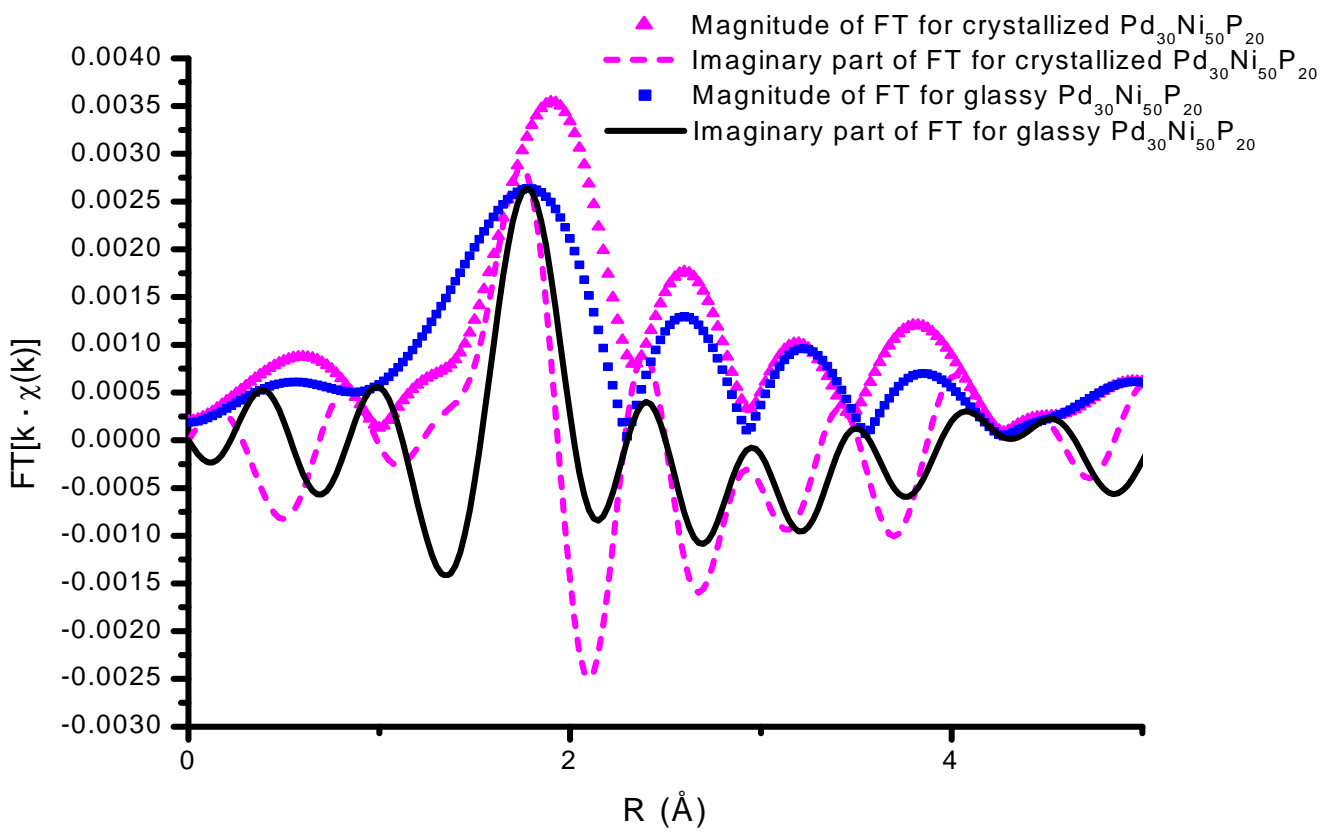

Figure 1: A phase change is observed in the nearest neighbor environment of $\mathrm{P}$ in $\mathrm{Pd}_{30} \mathrm{Ni}_{50} \mathrm{P}_{20}$ during crystallization. This is due to a change of the $\mathrm{P}$ nearest neighbors from $\mathrm{Ni}$ to $\mathrm{Pd}$ ones
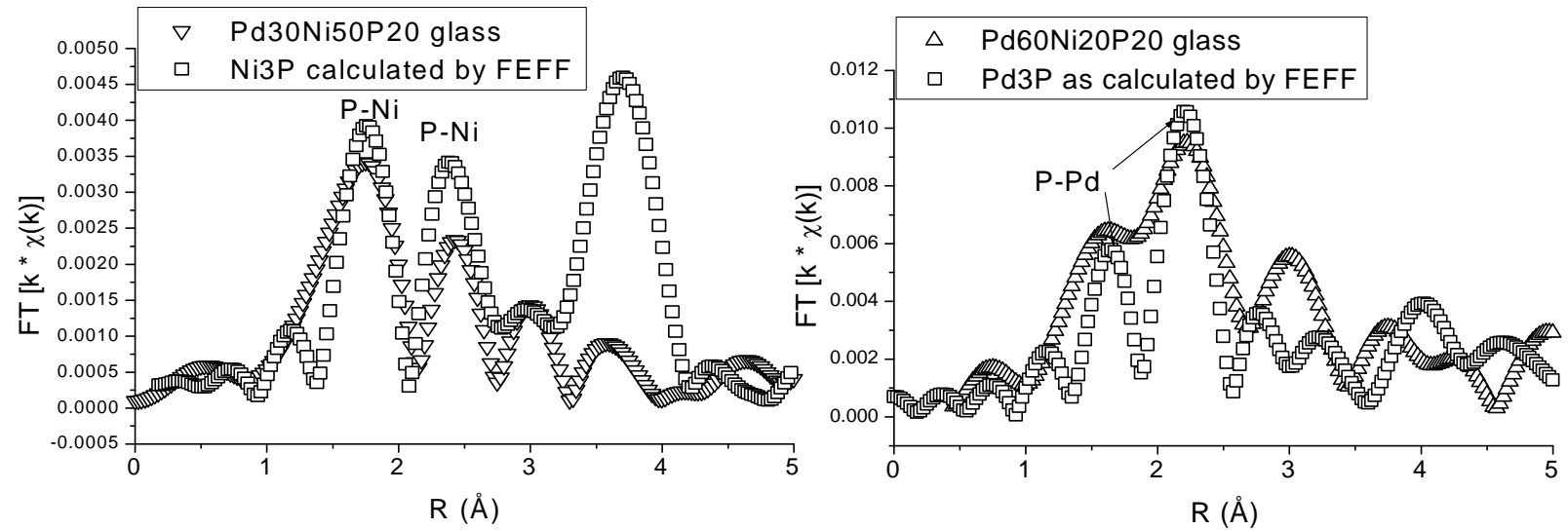

Figure 2: $\mathrm{P}$ nn environments in $\mathrm{Pd}_{30} \mathrm{Ni}_{50} \mathrm{P}_{20}$ and $\mathrm{Pd}_{60} \mathrm{Ni}_{20} \mathrm{P}_{20}$ glasses and those calculated for the structures of $\mathrm{Ni}_{3} \mathrm{P}$ and $\mathrm{Pd}_{3} \mathrm{P}$ crystals, respectively, using FEFF7. The spectrum of $\mathrm{Pd}_{60} \mathrm{Ni}_{20} \mathrm{P}_{20}$ has been shifted by $0.48 \AA$ to correct for phase shift as calculated by FEFF7. 